\title{
Connexin 43-autophagy loop in the podocyte injury of diabetic nephropathy
}

\author{
JIALING JI $^{1 *}$, YAJIE ZHAO $^{1 *}$, CHEN NA $^{2 *}$, MIN YANG $^{3}$, XIANYI ZHU ${ }^{1}$, \\ HUIMIN SHI ${ }^{1}$, WEIHUA GAN ${ }^{1}$ and AIQING ZHANG ${ }^{1}$ \\ ${ }^{1}$ Department of Pediatric Nephrology, 2nd Affiliated Hospital of Nanjing Medical University, Nanjing, Jiangsu 210003; \\ ${ }^{2}$ Department of Pediatrics, Yanan Hospital Affiliated to Kunming Medical University, Kunming, Yunnan 650000; \\ ${ }^{3}$ Department of Nephrology, Comprehensive Laboratory, The Third Affiliated Hospital of Soochow University, \\ Changzhou, Jiangsu 213000, P.R. China
}

Received March 16, 2019; Accepted August 23, 2019

DOI: $10.3892 / \mathrm{ijmm} .2019 .4335$

\begin{abstract}
The reduction of podocyte injury is a key strategy in controlling proteinuria, which is the main early clinical manifestation of diabetic nephropathy (DN). Impaired autophagic flux is the primary mechanism responsible for podocyte injury in DN. The aim of the present study was to elucidate the effect of connexin 43 (Cx43) on impaired autophagic flux in podocyte injury and to explore its molecular mechanism of action in DN. Sprague-Dawley rats were administered streptozocin (STZ) to construct a DN animal model. Podocytes were incubated in media containing either buffer or high glucose $(\mathrm{HG}$; $30 \mathrm{mM}$ ) for variable time periods. The podocytes were then examined and the mechanism of injury was investigated using an Annexin V/PI assay, immunofluorescence staining, western blotting, and RNA interference. In vivo, STZ-induced DN rats with or without $\mathrm{Cx} 43$ knockdown were established to observe the role of $\mathrm{Cx} 43$ in autophagic flux and podocyte injury. We observed that HG induced podocyte injury, accompanied by increases in Cx43 expression and impaired autophagic flux, as evidenced by the accumulation of LC3II/LC3I and p62. Interestingly, the silencing of $\mathrm{Cx} 43$ expression ameliorated autophagic flux impairment and reduced podocyte injury via suppression of the mammalian target of rapamycin pathway. Furthermore, impaired autophagic flux also blocked the degradation of $\mathrm{Cx} 43$. In vitro studies indicated that higher numbers of Annexin V/PI-positive podocytes, impaired autophagic flux
\end{abstract}

Correspondence to: Dr Weihua Gan or Dr Aiqing Zhang, Department of Pediatric Nephrology, 2nd Affiliated Hospital of Nanjing Medical University, 262 Zhongshanbeilu, Nanjing, Jiangsu 210003, P.R. China

E-mail: weihuagan@njmu.edu.cn

E-mail: njaiqing@njmu.edu.cn

*Contributed equally

Key words: connexin 43, autophagy, podocyte injury, diabetic nephropathy, mammalian target of rapamycin signaling pathway and increased $\mathrm{Cx} 43$ expression were observed in $\mathrm{HG}$-induced podocyte injury relative to the control group. The pathogenic effect of Cx43 on impaired autophagic flux and podocyte injury was also confirmed by $\mathrm{Cx} 43$ knockdown. The present study provided preliminary evidence indicating that the interdependence of Cx43 and impaired autophagic flux represents a novel mechanism of podocyte injury in DN. Hence, the $\mathrm{Cx} 43$-autophagy loop is a potentially relevant therapeutic target for the treatment of DN.

\section{Introduction}

Diabetic nephropathy (DN) is a leading cause of chronic kidney disease (CKD) and end-stage renal failure, constituting a major health concern worldwide (1). Damage to the glomerular filtration unit is one of the most important pathological characteristics of DN. The filtration unit comprises capillary endothelial cells, the glomerular basement membrane and podocytes, which are specialized epithelial cells. Once considered to be primarily quiescent and terminally differentiated cells, podocytes have been identified as a key factor in DN (2). Podocyte injury is characterized by the pathological loss of regularity in branching and widening of the foot processes, termed 'foot process effacement'. Severe insults lead to podocyte loss by apoptosis or detachment. Numerous studies have reported that disruption of multiple renal signaling pathways is crucial for the progression of such pathological damage $(3,4)$. However, there are currently no effective therapeutic options for DN in the clinical setting. Indeed, the precise regulatory signaling pathways through which podocytes are injured in DN are largely unclear.

Autophagy is a major catabolic pathway through which mammalian cells degrade and recycle macromolecules and organelles. The major roles of autophagy are the removal of damaged organelles, degradation of proteins, and reconstitution of intracellular metabolism for the maintenance of intracellular homeostasis and cell health (5). Generally, autophagy is upregulated under conditions of oxidative stress and hypoxia in podocytes. Recently, convincing evidence indicates that, in the case of diabetes, autophagy plays a pivotal role in maintaining the homeostasis of lysosomes in 
podocytes. The impairment of autophagy, as it is implicated in the pathogenesis of podocyte loss, may lead to massive overflow proteinuria in DN (6-8). However, the exact role of autophagy in podocytes remains elusive.

Connexins (Cxs) are a multigenic family of transmembrane proteins that form gap junctions and participate in the exchange of information between cells. Connexin 43 (Cx43) is considered to be the most abundant and widely expressed gap junction protein. Extensive studies have revealed that, in addition to its role in intercellular communication, Cx43 mediates gene transcription, cytoskeleton dynamics, ATP exocytosis, vesicle release and cell stress (9). Sawai et al (10) reported the presence of $\mathrm{Cx} 43$ in normal podocytes in a linear pattern, and demonstrated a shift in this linear distribution in patients with DN. Our previous studies also indicated that upregulation of $\mathrm{Cx} 43$ is involved in podocyte injury (11), suggesting that $\mathrm{Cx} 43$ may be a critical regulator in podocytes under DN conditions. Furthermore, Cx43 has recently been implicated in inflammation and fibrosis. Inhibiting Cx43 may alleviate kidney damage and maintain renal function. Therefore, new therapies targeting Cx43 blockade in ideal cell populations may be a viable option for effectively inhibiting the progression of CKD (12). Interestingly, Cx43 rapidly modulates autophagy response, playing a critical role in cell apoptosis (13). However, the effect of $\mathrm{Cx} 43$ on the regulation of podocyte autophagy under DN conditions remains unclear.

The aim of the present study was to determine the effect of Cx43 on impaired autophagic flux, and to determine whether the regulation of $\mathrm{Cx} 43$ can protect podocytes under DN conditions.

\section{Materials and methods}

Antibodies and reagents. Rapamycin (RP) and chloroquine (CQ) were purchased from Sigma-Aldrich; Merck KGaA. Antibodies against LC3, mammalian target of rapamycin (mTOR) and p-mTOR were acquired from Cell Signaling Technology, Inc. Anti-Cx43, anti-podocin, anti-nephrin and anti-p62 antibodies were obtained from Abcam. Anti-GAPDH was purchased from CWBio.

Animals. The study protocols were reviewed and approved by the Institutional Animal Care and Use Committee of Nanjing Medical University. A total of 24 male Sprague-Dawley rats (aged 5-6 weeks and weighing 190 g) were housed under specific pathogen-free conditions at optimal temperature with a 12-h light/dark cycle, and were allowed free access to standard food and water. The rats were randomly divided into four groups: Group 1, PBS-infused rats (control, $n=6$ ); group 2, streptozocin (STZ; $60 \mathrm{mg} / \mathrm{kg})$-infused rats $(\mathrm{n}=6)$; group 3, STZ (60 mg/kg)-infused rats with scrambled siRNA (SCR, n=6); and group 4, STZ (60 mg/kg)-infused rats with Cx43 siRNA [oligodeoxynucleotide antisense (AS), n=6]. At the end of the 28-day infusion period, the rats were weighed and blood and urine samples were collected. The blood urea nitrogen and urine protein levels were analyzed according to the manufacturer's protocol (R\&D Systems, Inc.). Tail capillary blood glucose levels were monitored with a glucometer (Accu-Chek Performa; Roche Diagnostics GmbH).
Cell culture. The immortalized mouse podocyte cell line MPC5 was kindly provided by Dr Junwei Yang (Nanjing Medical University) and the cells were cultured as previously described (14). Podocytes were differentiated without interferon- $\gamma$ at $37^{\circ} \mathrm{C}$ for 14 days prior to the experiments. Differentiated podocytes were incubated in medium containing $0.1 \%$ fetal bovine serum for $24 \mathrm{~h}$. The podocytes exposed to $\mathrm{HG}(30 \mathrm{mM})$ were then cultured for various time periods.

Transfection of small interference RNA. Podocytes were transfected with CX43 siRNA (50 nM) (sense, 5'-AAAGUU GCUGCUGGACAUGAATT-3' and antisense, 5'-UUCAUG UCCAGCAGCAACUUUTT-3') or negative control siRNA (sense, 5'-UUCUCCGAACGUGUCACGUTT-3' and antisense, 5'-ACGUGACACGUUCGGAGAATT-3') for $24 \mathrm{~h}$ using Lipofectamine ${ }^{\circledR} 3000$ (Invitrogen; Thermo Fisher Scientific, Inc.) according to the manufacturer's protocol. Thereafter, the level of targeting protein with knockdown of $\mathrm{Cx} 43$ was detected by western blotting.

Western blotting. The cells were harvested after treatment with the different compounds for the indicated times. Protein levels were detected by western blotting according to established protocols (15). Primary antibodies against Cx43 (1:1,000), LC3 (1:1,000), p62 (1:2,000), podocin $(1: 1,000)$, synaptopodin $(1: 1,000)$, mTOR $(1: 1,000), \mathrm{p}-\mathrm{mTOR}(1: 1,000)$ and GAPDH $(1: 2,000)$ were used.

Annexin V-fluorescein isothiocyanate conjugated with propidium iodide (PI) staining. Podocyte injury was quantified by Annexin V/PI staining (BD Biosciences) following the manufacturer's protocol. Briefly, cells were harvested and washed twice with PBS. Subsequently, the cells were resuspended in $100 \mu \mathrm{l}$ binding buffer, then incubated with $5 \mu \mathrm{l}$ Annexin $\mathrm{V}$ and $10 \mu \mathrm{PI}$ for $15 \mathrm{~min}$ at $25^{\circ} \mathrm{C}$ in the dark. After mixing gently in $400 \mu \mathrm{l}$ of binding buffer, the cells were observed under a fluorescence microscope (DS-Ri1; Nikon Corporation).

Immunofluorescence staining. Paraffin sections (3- $\mu \mathrm{m})$ were prepared and incubated with primary antibodies against synaptopodin, Wilms' tumor-1 (WT-1) and p-mTOR in PBS containing $1 \% \mathrm{BSA}$ overnight at $4^{\circ} \mathrm{C}$ after blocking with $5 \%$ BSA for $1 \mathrm{~h}$. Subsequently, the sections were incubated with secondary antibody in PBS containing $1 \%$ BSA in the dark. After washing with PBS, cell nuclei were stained with DAPI. Immunostained samples were visualized under a fluorescence microscope (Nikon Corporation).

Immunohistochemistry. Paraffin-embedded sections were used for immunohistochemistry. Briefly, the sections were incubated with primary antibodies against $\mathrm{Cx} 43$ and podocin overnight at $4^{\circ} \mathrm{C}$. The sections were then analyzed using a streptavidin peroxidase detection system (Maixin) according to the manufacturer's protocol. Reactions were conducted using a DAB substrate kit (Maixin) and counterstaining was performed using hematoxylin. The sections were visualized under a microscope (Nikon Corporation).

Statistical analysis. Data are presented as the mean \pm the standard error of the mean. When more than two groups 

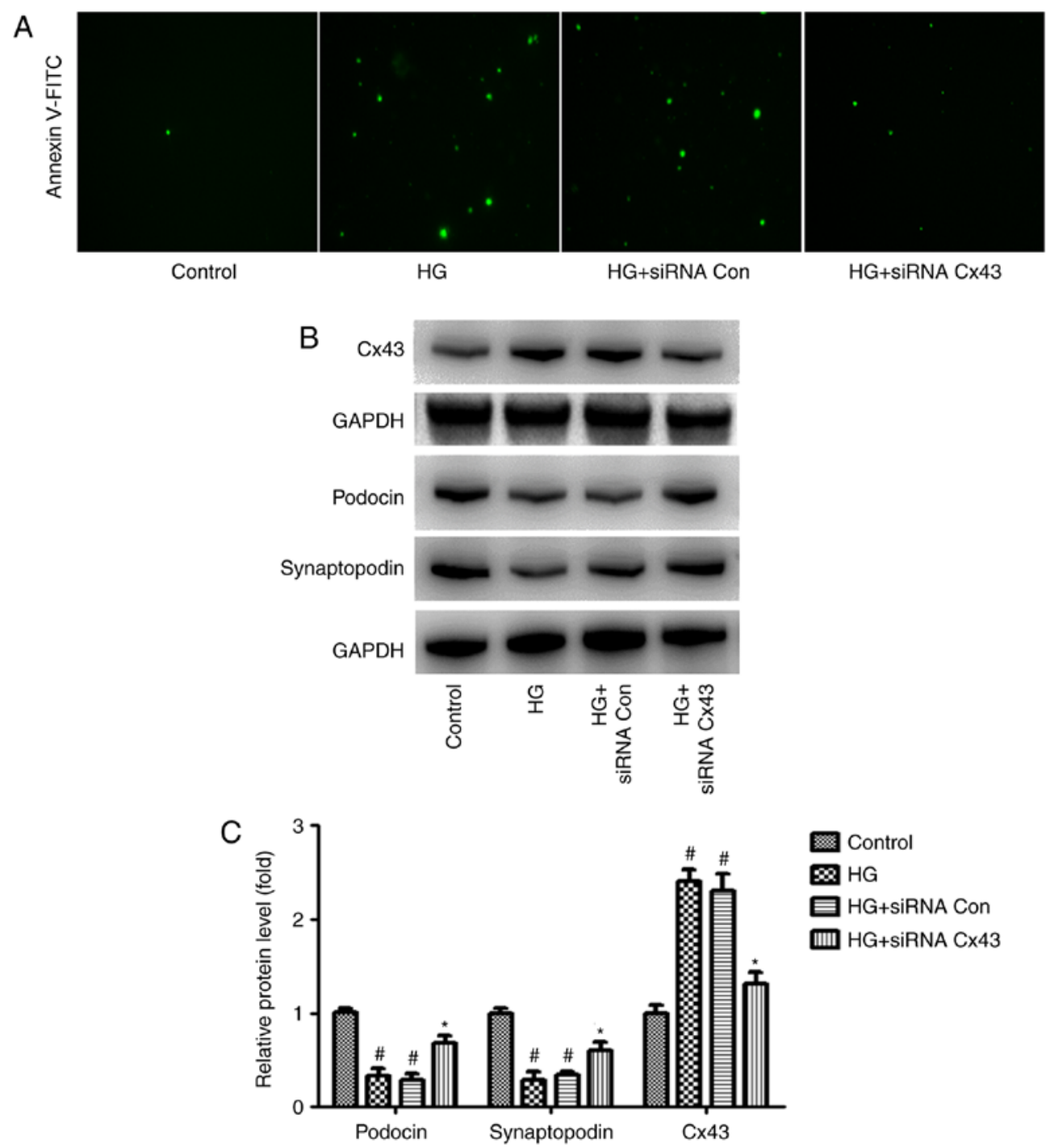

Figure 1. Effect of $\mathrm{Cx} 43$ on podocyte apoptosis. Podocytes were transfected with $\mathrm{Cx} 43$ siRNA $(60 \mathrm{nM})$ or siRNA NC and cultured in HG conditions for $24 \mathrm{~h}$. (A) Annexin V-FITC-labeled injured podocytes viewed under a fluorescence microscope. Magnification, x200. (B) Representative western blots of Cx43, podocin and synaptopodin and (C) quantification of western blotting. GAPDH levels were used as standard loading controls (mean \pm standard error of the mean, $\mathrm{n}=3$ ). ${ }^{\text {"P}} \mathrm{P}<0.05$ vs. control group; ${ }^{*} \mathrm{P}<0.05$ vs. $\mathrm{HG}+$ siRNA control group (Bonferroni's correction). HG, high glucose.

were compared, one-way analysis of variance followed by Bonferroni's correction was employed to analyze the differences using SPSS 22.0 statistical software (IBM Corp.). A two-sided P-value of $<0.05$ was considered to indicate statistically significant differences.

\section{Results}

CX43 silencing attenuates $H G$-induced podocyte injury. It was observed that podocyte injury increased significantly in the HG group, as did the expression of $\mathrm{Cx} 43$, suggesting that abnormal activation of $\mathrm{Cx} 43$ may be involved in HG-induced podocyte injury. To examine this hypothesis, $\mathrm{Cx} 43$ function was inhibited with Cx43 siRNA in the podocytes. siRNA-control served as a negative control. Interestingly, following transfection with Cx43 siRNA, we observed that, compared with the siRNA-control group, podocyte injury was markedly attenuated, as evidenced by the number of Annexin V/PI-positive cells (green) (Fig. 1A). In addition, increased levels of podocin and synaptopodin (surrogate markers for podocytes) were observed in podocytes transfected with Cx43 siRNA via western blot analysis (Fig. 1B). These findings indicate that the inhibition of $\mathrm{Cx} 43$ plays a protective role against $\mathrm{HG}$-induced podocyte injury.

Cx43 negatively regulates autophagic flux by enhancing mTOR signaling in HG-induced podocytes. In addition, impaired autophagic flux was also observed in HG-induced podocytes, as demonstrated by the increased expression levels of p62 and LC3II/LC3I. Convincing evidence has demonstrated that connexins play a key role in autophagosome formation (16). To examine whether $\mathrm{Cx} 43$ regulates autophagic flux in HG-induced podocytes, Cx43 siRNA was also used to inhibit $\mathrm{Cx} 43$ expression. Of note, compared with podocytes transfected with NC-siRNA, the LC3II/LC3I ratio and the level of p62 expression were markedly decreased in Cx43 siRNA-treated podocytes, as detected by western blotting, indicating that $\mathrm{Cx} 43$ also plays a key role in regulating autophagic flux. Subsequently, the exact mechanism of the regulatory role of $\mathrm{Cx} 43$ on autophagic flux was investigated. 

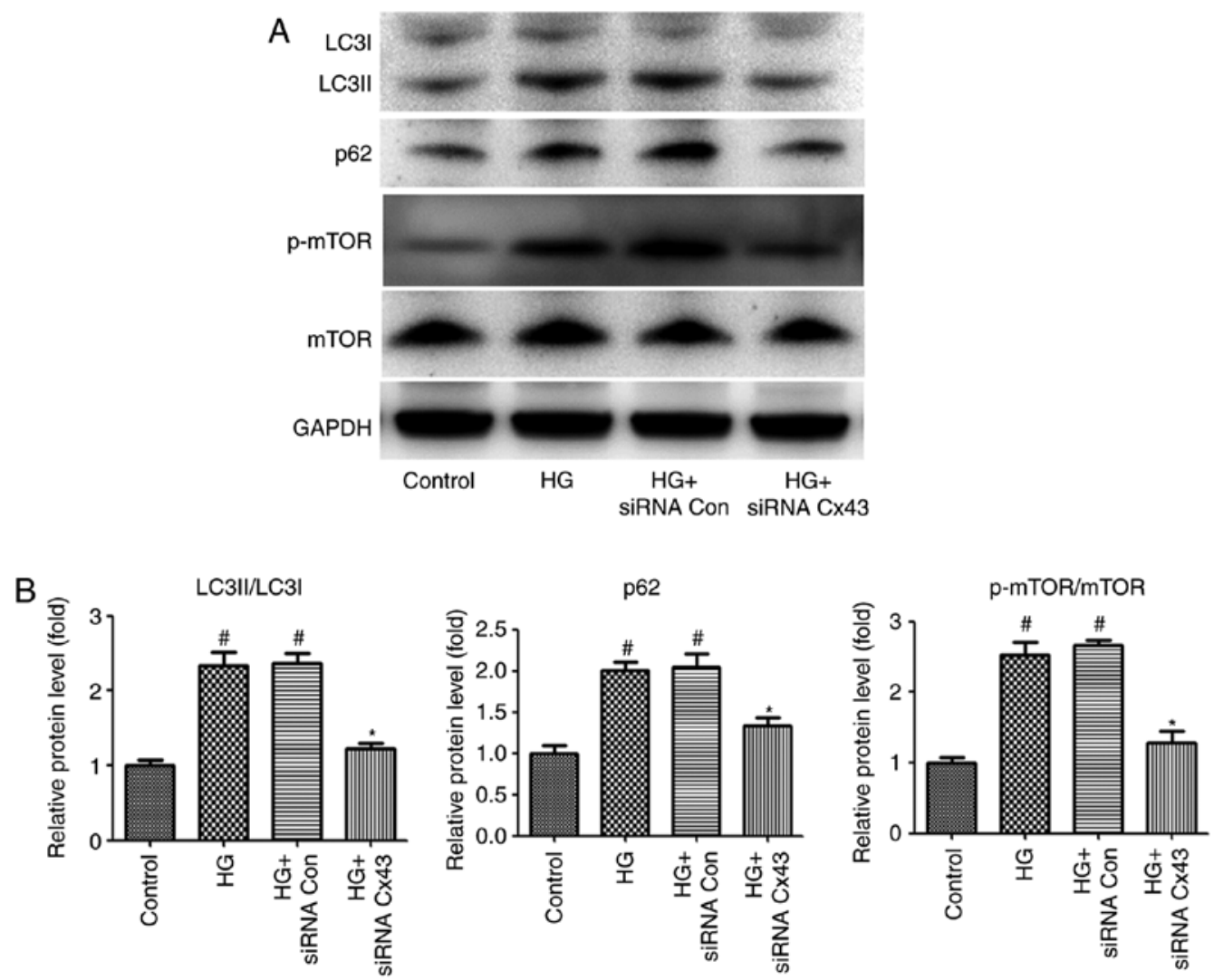

Figure 2. Regulation of autophagic flux by activating the mTOR pathway. (A) Western blotting of LC3, p62, p-mTOR and mTOR in HG-induced podocytes transfected with Cx43 siRNA (50 nM) or siRNA NC. (B) The quantitative ratios are shown as relative optical densities of bands that are normalized to the expression of GAPDH. The data are presented as mean \pm standard error of the mean; $n=3$ per group; ${ }^{*} \mathrm{P}<0.05$ vs. control group; ${ }^{*} \mathrm{P}<0.05$ vs. HG group (Bonferroni's correction). mTOR, mammalian target of rapamycin; HG, high glucose.

Accumulating evidence has demonstrated that mTOR signaling is a pharmacological target for autophagy regulation (17). Therefore, attention was focused on mTOR signaling. The results revealed that the $\mathrm{p}-\mathrm{mTOR} / \mathrm{mTOR}$ protein ratio decreased significantly in the $\mathrm{Cx} 43$ siRNA group $(\mathrm{P}<0.05$; Fig. 2), confirming that $\mathrm{Cx} 43$ inhibited autophagy by activating the mTOR signaling pathway in HG-induced podocytes.

Impaired autophagic flux blocks the degradation of Cx43 in HG-induced podocytes. Accumulating evidence demonstrates that $\mathrm{Cx} 43$ is also regulated by autophagy under certain pathological conditions $(18,19)$, suggesting that autophagy may regulate $\mathrm{Cx} 43$ expression in HG-induced podocytes. To examine this hypothesis, CQ and RP, acting as an autophagy inhibitor and an autophagy inducer, respectively $(20,21)$, were used to regulate autophagic flux. Interestingly, compared with the HG group, the level of $\mathrm{Cx} 43$ increased significantly in the HG + CQ group $(\mathrm{P}<0.05)$. By contrast, the level of $\mathrm{Cx} 43$ decreased significantly in the $\mathrm{HG}+\mathrm{RP}$ group compared with the HG group $(\mathrm{P}<0.05$; Fig. 3$)$. These results indicated that $\mathrm{Cx} 43$ activation is also regulated by impaired autophagic flux in HG-induced podocytes.

Cx43 silencing improves autophagic flux in the glomeruli of $D N$ rats. To determine the effect of $\mathrm{Cx} 43$ on autophagic flux in the glomeruli in a DN model in vivo, $\mathrm{Cx} 43$ siRNA or scrambled negative control siRNA was administered to the rats via the tail vein. After induction of diabetes, the DN rats and Cx43 siRNA-treated DN rats were compared in terms of body weight, blood urea nitrogen (BUN), urine protein and blood glucose levels. Of note, body weight was higher while blood glucose, urine protein and BUN levels were lower in Cx43 siRNA-treated diabetic rats compared with their control diabetic littermates (Table I). Immunohistochemical analysis revealed that $\mathrm{Cx} 43$ siRNA treatment significantly reduced Cx43 expression in the glomeruli (Fig. 4A). Interestingly, compared with scrambled negative control-injected mice, $\mathrm{Cx} 43$ siRNA administration efficiently reversed the enhanced autophagic flux, as detected by western blotting (Fig. 4B). Thus, the inhibition of $\mathrm{Cx} 43$ attenuated the enhanced autophagic flux in the glomeruli of rats with DN.

Cx43 negatively regulates autophagic flux by enhancing mTOR signaling in the glomeruli of DN rats. To examine whether mTOR signaling is involved in the regulatory effect of $\mathrm{Cx} 43$ on autophagic flux in vivo, mTOR levels were measured. Consistently with the results in vitro, immunofluorescence analysis revealed that the number of p-mTOR-positive cells decreased in the glomeruli of DN rats following treatment with $\mathrm{Cx} 43$ siRNA (Fig. 5A). Furthermore, the p-mTOR/mTOR protein ratio was markedly decreased, as confirmed by western blotting (Fig. 5B). Therefore, activation of the mTOR pathway may negatively contribute to $\mathrm{Cx} 43$-regulated autophagic flux.

Cx43 silencing attenuates podocyte injury in DN rats. To determine the role of $\mathrm{Cx} 43$ in podocyte injury in vivo, 
Table I. Comparison of body weight, BUN, urine protein and blood glucose levels among different rat groups after induction of diabetes.

\begin{tabular}{lcccc}
\hline Groups & Body weight $(\mathrm{g})$ & Blood glucose $(\mathrm{mmol} / \mathrm{l})$ & BUN (mmol/l) & Urine protein (mg/24 h) \\
\hline Control & $501.6 \pm 14.1$ & $6.6 \pm 0.3$ & $4.6 \pm 0.6$ & $8.2 \pm 0.5$ \\
DN & $310.5 \pm 7.9^{\mathrm{a}}$ & $30.5 \pm 2.3^{\mathrm{a}}$ & $9.7 \pm 1.3^{\mathrm{a}}$ & $24.6 \pm 4.3^{\mathrm{a}}$ \\
DN + Cx43 SCR & $292.3 \pm 7.3^{\mathrm{a}}$ & $29.8 \pm 2.1^{\mathrm{a}}$ & $8.7 \pm 1.1^{\mathrm{a}}$ & $25.8 \pm 5.1^{\mathrm{a}}$ \\
DN + Cx43 AS & $433.5 \pm 8.5^{\mathrm{b}}$ & $28.7 \pm 2.3$ & $5.6 \pm 0.7^{\mathrm{b}}$ & $12.8 \pm 3.1^{\mathrm{b}}$ \\
\hline
\end{tabular}

${ }^{\mathrm{a}} \mathrm{P}<0.05$ vs. control group; ${ }^{\mathrm{b}} \mathrm{P}<0.05$ vs. $\mathrm{DN}$ group. $\mathrm{n}=3$ per group (Bonferroni's correction). BUN, blood urea nitrogen; DN, diabetic nephropathy.
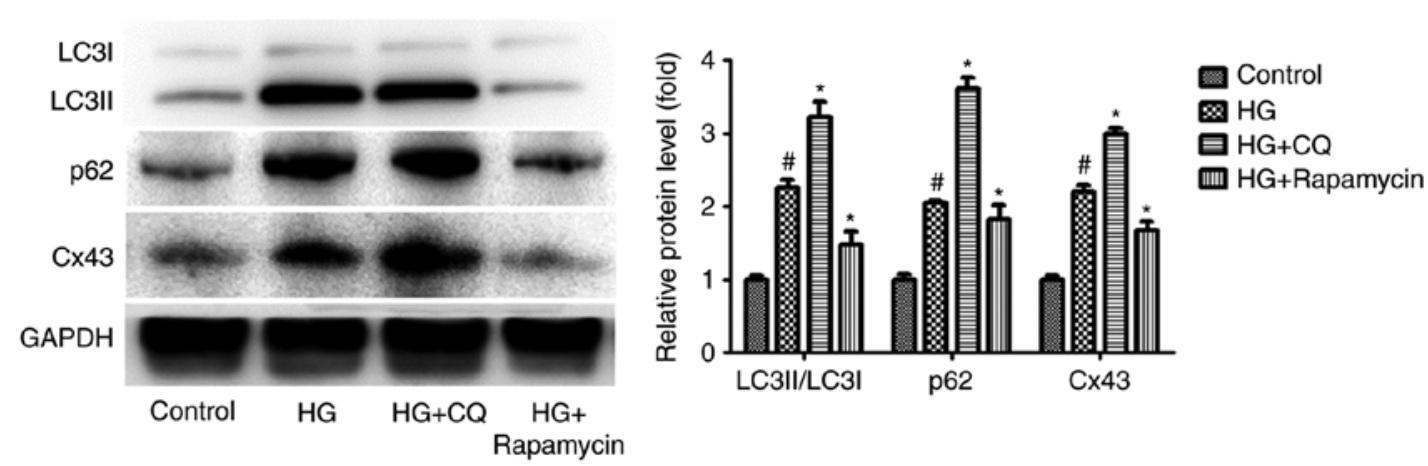

Figure 3. Impaired autophagic flux and Cx43 activation in HG-induced podocytes. The podocytes pretreated with CQ (10 nM) or RP (1 nM) were cultured in high glucose $(\mathrm{HG} ; 30 \mu \mathrm{M})$ for $24 \mathrm{~h}$. Representative western blots of LC3II/LC3I, p62 and Cx43 and quantification of western blotting. GAPDH levels were used as standard loading controls (mean \pm standard error of the mean, $\mathrm{n}=3$ ). ${ }^{*} \mathrm{P}<0.05$ vs. control group; ${ }^{*} \mathrm{P}<0.05$ vs. HG group (Bonferroni's correction). $\mathrm{HG}$, high glucose; $\mathrm{CQ}$, chloroquine; RP, rapamycin.

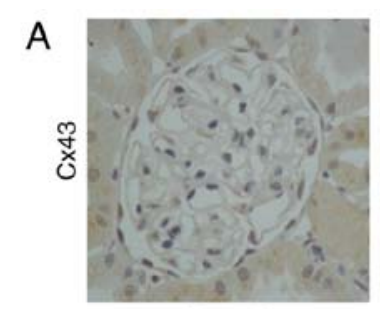

Control

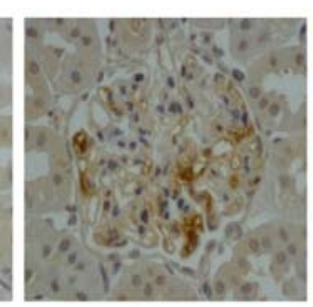

DN

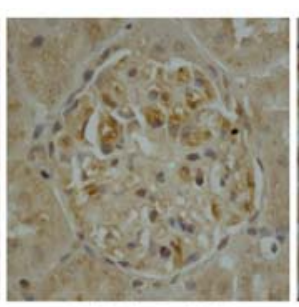

$\mathrm{DN}+\mathrm{C} \times 43 \mathrm{SCR}$

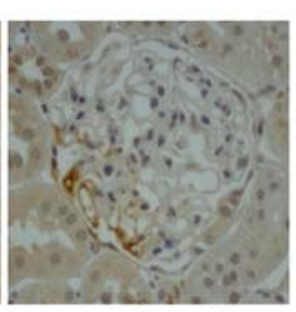

$\mathrm{DN}+\mathrm{C} \times 43 \mathrm{AS}$
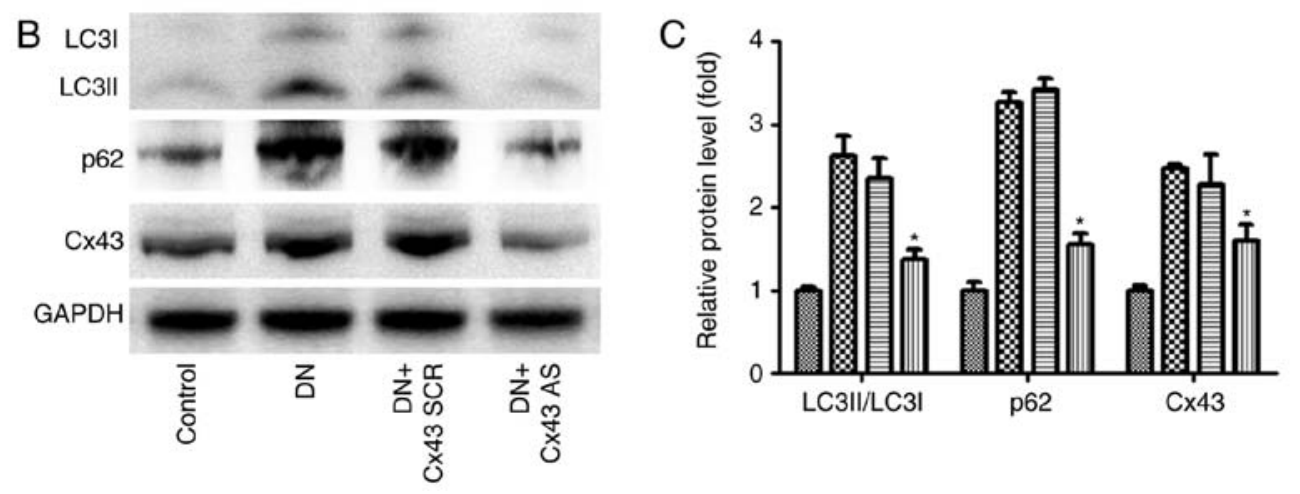

Figure 4. Effect of Cx43 silencing on podocyte autophagy. The rats were injected with STZ, followed by placement of an osmotic minipump with which to infuse $\mathrm{Cx} 43$ siRNA $(50 \mu \mathrm{M})$ or NC-siRNA $(50 \mu \mathrm{M})$ for 28 days. (A) Kidney tissue sections were subjected to histological examination via immunohistochemistry (Cx43, magnification, x400). (B and C) Western blot analysis of LC3, p62 and Cx43. The data are presented after normalization to GAPDH expression and quantified as mean \pm standard error of the mean; $\mathrm{n}=3$ per group; ${ }^{*} \mathrm{P}<0.05$ vs. control group; ${ }^{*} \mathrm{P}<0.05$ vs. DN group (Bonferroni's correction). STZ, streptozocin; DN, diabetic nephropathy.

immunostaining for WT-1 and synaptopodin was performed. The results revealed that the number of podocytes was significantly increased following treatment with Cx43 siRNA $(\mathrm{P}<0.05$; Fig. 6A and B). Similarly, compared with the 


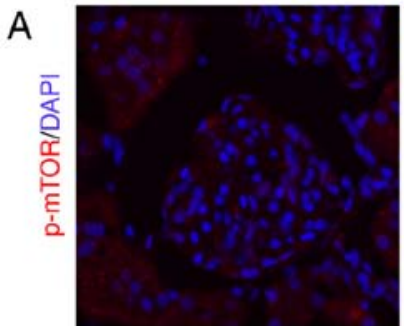

Control

\section{B}

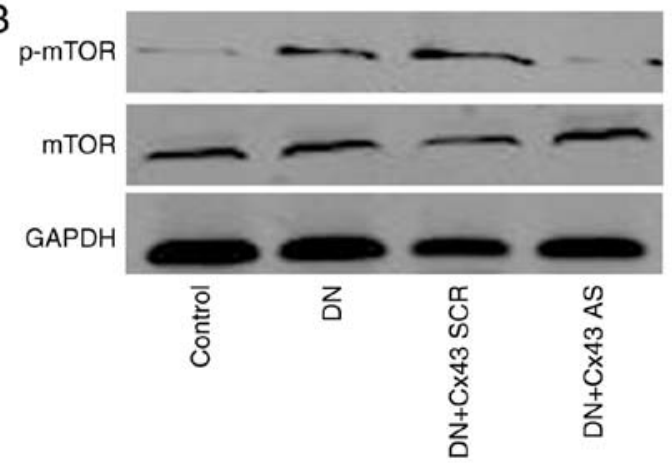

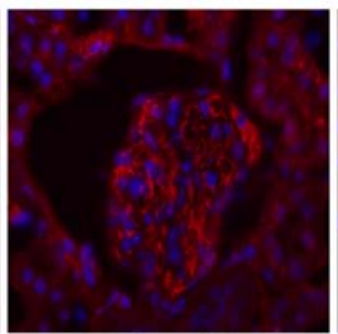

$\mathrm{DN}+\mathrm{C} \times 43 \mathrm{SCR}$

C

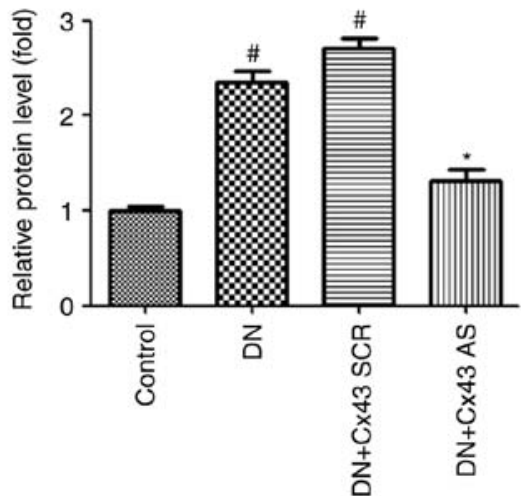

Figure 5. Cx43 negatively regulated autophagic flux by enhancing mTOR signaling in the glomeruli of a DN rat model. (A) Immunofluorescence results (magnification, $\mathrm{x} 400$ ) indicating the expression of p-mTOR in each group. Blue, nuclear staining (DAPI); green, p-mTOR staining. (B) Western blot analysis of p-mTOR and mTOR. (C) Relative protein level of p-mTOR/mTOR in the control, DN, DN + Cx43 SCR, DN + Cx43 AS groups. The data are presented as the mean \pm standard error of the mean, $n=3$ per group; ${ }^{*} \mathrm{P}<0.05$ vs. control group; ${ }^{\mathrm{P}}<0.05$ vs. $\mathrm{DN}$ group (Bonferroni's correction). $\mathrm{DN}$, diabetic nephropathy; mTOR, mammalian target of rapamycin.

A

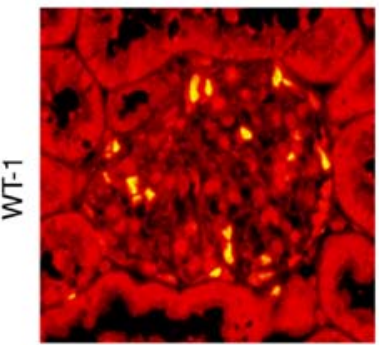

B

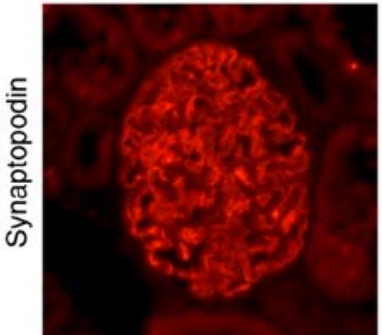

C

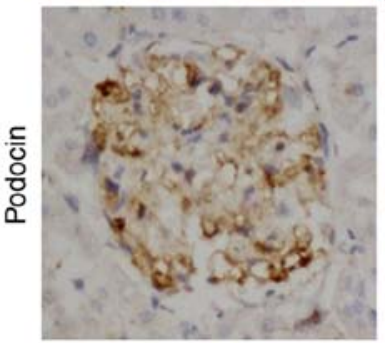

Control
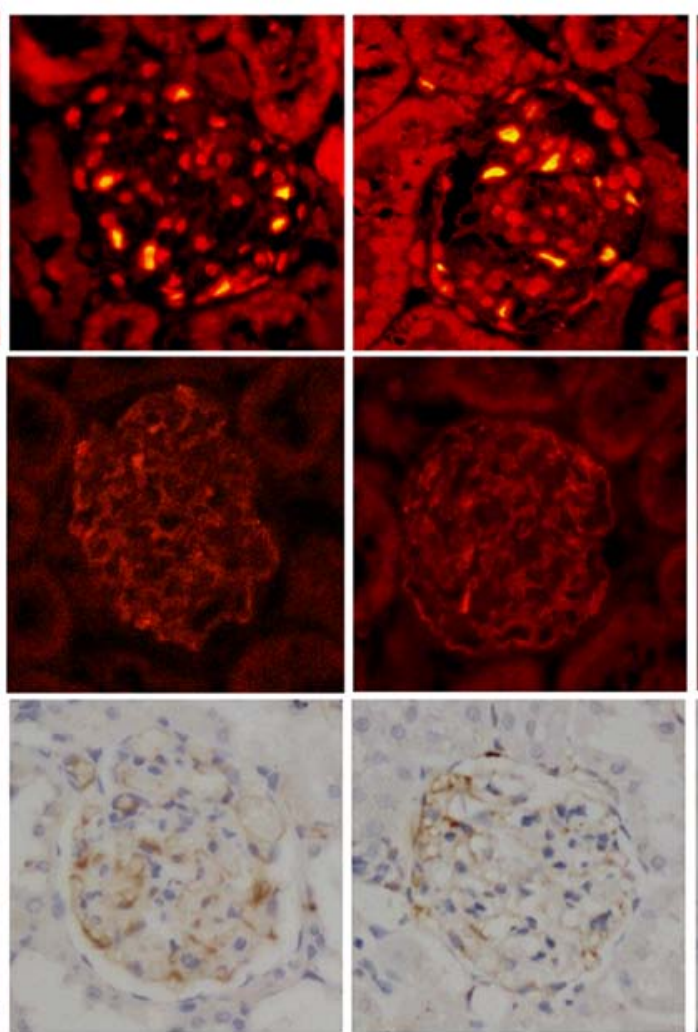

DN

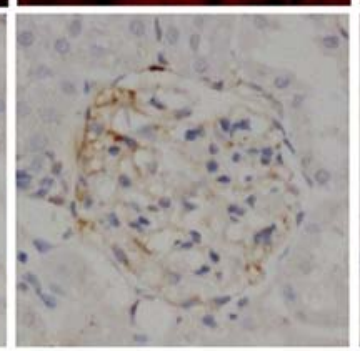

$\mathrm{DN}+\mathrm{C} \times 43 \mathrm{SCR}$
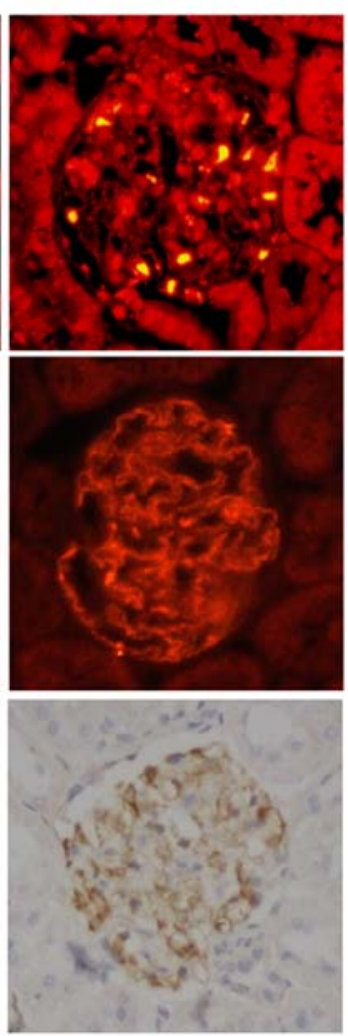

$\mathrm{DN}+\mathrm{C} \times 43 \mathrm{AS}$

Figure 6. Silencing of Cx43 improved podocyte injury in DN rat model. (A and B) Representative images of WT-1 and synaptopodin (surrogate markers for podocyte number) immunostaining in kidney sections from DN, DN + Cx43 SCR, DN + Cx43 AS and control groups (magnification, $\mathrm{x} 400$ ). (C) Kidney tissue sections were subjected to histological examination by immunohistochemistry to evaluate podocyte injury (podocin; magnification, $\mathrm{x} 400$ ). DN, diabetic nephropathy; WT-1, Wilms' tumor-1. 
NC-siRNA-treated DN rats, podocin (a marker for podocytes) also increased after knockdown of $\mathrm{Cx} 43$, as assessed by immunohistochemical staining analysis (Fig. 6C). Thus, Cx43 activation appears to promote podocyte injury.

\section{Discussion}

The critical role of podocyte injury, the characteristic pathological change of DN, in the initiation and progression of DN has been recognized for several years, and impaired autophagy appears to play a key role in podocyte injury $(22,23)$. It was recently reported that $\mathrm{Cx} 43$ is involved in the autophagic process (24). However, whether Cx43 plays a critical role in podocyte autophagy in DN remains largely unknown. The present study demonstrated the involvement of increases in $\mathrm{Cx} 43$ expression and impaired autophagic flux in HG-induced podocyte injury. The silencing of Cx43 expression ameliorated impaired autophagic flux and reduced podocyte injury by suppressing the mTOR pathway. In vivo studies indicated that the inhibition of $\mathrm{Cx} 43$ improved impaired autophagic flux in STZ-induced DN animal models. Furthermore, the pathogenic effect of $\mathrm{Cx} 43$ on podocyte injury was also confirmed. These findings may facilitate the identification of novel therapeutic targets for the treatment of podocyte injury in DN.

Autophagy ('self-eating') is a tightly regulated process that delivers senescent intracellular constituents to lysosomes for degradation in order to maintain intracellular homeostasis. An emerging body of research demonstrated that autophagy regulation is closely linked to human health $(25,26)$. Consistently with previous studies on aldosterone-induced podocyte injury (27), we herein observed that impaired autophagic flux was involved in HG-induced podocyte injury. Furthermore, increased $\mathrm{Cx} 43$ expression was also observed in association with this pathogenic process. However, whether Cx43 plays a critical role in impaired autophagic flux remains elusive.

Accumulating evidence has demonstrated that $\mathrm{Cx} 43$ may be involved in the process of podocyte injury in various kidney diseases $(10,11,28)$. Our in vitro and in vivo data demonstrated that the expression of $\mathrm{Cx} 43$ was also elevated by treatment with $\mathrm{HG}$, which may facilitate podocyte injury. Interestingly, after silencing Cx43 expression, HG-induced injury in cultured podocytes was partially attenuated by improving impaired autophagic flux, suggesting that $\mathrm{Cx} 43$ was partially involved in HG-induced podocyte injury and that the amelioration of impaired autophagic flux represents a novel mechanism mediating the effect of $\mathrm{Cx} 43$ on podocyte injury. However, the exact regulatory mechanisms of Cx43 on impaired autophagic flux have yet to be fully elucidated.

mTOR is a well-known master regulator of cellular metabolism, growth and proliferation in response to a wide range of triggers. The deregulation of mTOR signaling, which plays a key role in the regulation of autophagy, has been implicated in a number of human diseases, including diabetes, neurodegenerative diseases and cancer $(29,30)$. Interestingly, in the present study, the mTOR signaling pathway acted as a critical regulator involved in $\mathrm{Cx} 43$ /autophagy-mediated podocyte injury in DN. It was observed that mTOR signaling was activated after stimulation with $\mathrm{HG}$ in vivo and in vitro, indicating that an mTOR-dependent mechanism may be involved in Cx43-mediated podocyte injury in DN, which is consistent with previous results $(31,32)$. Furthermore, after inhibition of Cx43 function by $\mathrm{Cx} 43$ siRNA, the $\mathrm{p}-\mathrm{mTOR} / \mathrm{mTOR}$ protein ratio decreased markedly, followed by a decrease in autophagic flux, indicating that $\mathrm{Cx} 43$ may regulate autophagy flux by negatively affecting mTOR signaling, which may be partially responsible for podocyte injury in DN.

In addition, $\mathrm{Cx} 43$ was found to be regulated by autophagic flux. It has been reported that the initiation of autophagy includes the formation of the phagophore, the initial sequestering compartment, which expands into an autophagosome. Completion of the autophagosome is followed by fusion with lysosomes and degradation of its contents, allowing complete flux, or flow, through the entire pathway. As RP promotes initiation of the autophagic flux, LC3I to LC3II turnover is increased in the glomeruli. Of note, LC3II is also a selective substrate of autophagy. Hence, LC3II degradation is blocked in the presence of CQ, which inhibits the acidification of organelles and, subsequently, autophagosome-lysosome fusion (33). This may explain why CQ treatment caused a greater accumulation of LC3II in the HG + CQ group when compared with the $\mathrm{HG}+\mathrm{RP}$ group in the present study (even though CQ is an inhibitor of autophagy). Accordingly, compared with the HG group, the level of Cx43 increased significantly in the $\mathrm{HG}+\mathrm{CQ}$ group. On the contrary, the level of Cx43 decreased significantly in the $\mathrm{HG}+\mathrm{RP}$ group, indicating that a negative feedback mechanism is involved in Cx43-modulated podocyte injury to maintain the homeostasis of podocytes.

In summary, the findings of the present study uncovered a previously unknown mechanism implicated in podocyte injury under DN conditions. It was confirmed that upregulation of Cx43 expression negatively regulates autophagy by activating the mTOR signaling pathway in DN, while inhibiting Cx43 expression improved impaired autophagic flow and reduced podocyte damage. Hence, the $\mathrm{Cx} 43$-autophagy loop may be used as a novel therapeutic target for the development of new drugs for DN.

\section{Acknowledgements}

Not applicable.

\section{Funding}

The present study was supported by the National Natural Science Foundation of China (grant. no. 81670650); the Natural Science Foundation of Jiangsu Province Grant (grant. nos. BK20161071 and BK20161599); the Jiangsu Province Women and Children Health Key Talents (grant. no. FRC201737); the Nanjing Medical Science and Technique Development Foundation (grant. no. QRX17106); and the Health Commission Foundation of Jiangsu Province (grant. no. H2017002).

\section{Availability of data and materials}

The datasets analyzed during the present study are available from the corresponding author on reasonable request. 


\section{Authors' contributions}

JJ, WG and AZ designed the study, performed the experiments, analyzed the results, and wrote and edited the manuscript. YZ, $\mathrm{CN}$ and MY performed the experiments and discussed the results. XZ and HS performed the experiments and analyzed the data. All authors read and approved the final manuscript.

\section{Ethics approval and consent to participate}

The experimental procedures in the present study were approved by Nanjing Medical University (Nanjing, China).

\section{Patient consent for publication}

Not applicable.

\section{Competing interests}

The authors declare that they have no competing interests.

\section{References}

1. Gnudi L, Coward RJ and Long DA: Diabetic nephropathy: Perspective on novel molecular mechanisms. Trends Endocrinol Metab 27: 820-830, 2016.

2. Susztak K, Raff AC, Schiffer M and Böttinger EP: Glucoseinduced reactive oxygen species cause apoptosis of podocytes and podocyte depletion at the onset of diabetic nephropathy. Diabetes 55: 225-233, 2006.

3. Madhusudhan T, Wang H, Dong W, Ghosh S, Bock F, Thangapandi VR, Ranjan S, Wolter J, Kohli S, Shahzad K, et al: Defective podocyte insulin signalling through $\mathrm{p} 85$-XBP1 promotes ATF6-dependent maladaptive ER-stress response in diabetic nephropathy. Nat Commun 6: 6496, 2015.

4. Long J, Badal SS, Ye Z, Wang Y, Ayanga BA, Galvan DL, Green NH, Chang BH, Overbeek PA and Danesh FR: Long noncoding RNA Tug1 regulates mitochondrial bioenergetics in diabetic nephropathy. J Clin Invest 126: 4205-4218, 2016.

5. Mizushima N and Komatsu M: Autophagy: Renovation of cells and tissues. Cell 147: 728-741, 2011.

6. Tagawa A, Yasuda M, Kume S, Yamahara K, Nakazawa J, Chin-Kanasaki M, Araki H, Araki S, Koya D, Asanuma K, et al: Impaired podocyte autophagy exacerbates proteinuria in diabetic nephropathy. Diabetes 65: 755-767, 2016.

7. Liu Y, Zhang J, Wang Y and Zeng X: Apelin involved in progression of diabetic nephropathy by inhibiting autophagy in podocytes. Cell Death Dis 8: e3006, 2017.

8. Kume S, Yamahara K, Yasuda M, Maegawa H and Koya D: Autophagy: Emerging therapeutic target for diabetic nephropathy. Semin Nephrol 34: 9-16, 2014.

9. Ribeiro-Rodrigues TM, Martins-Marques T, Morel S, Kwak BR and Girão H: Role of connexin 43 in different forms of intercellular communication-gap junctions, extracellular vesicles and tunneling nanotubes. J Cell Sci 130: 3619-3630, 2017.

10. Sawai K, Mukoyama M, Mori K, Yokoi H, Koshikawa M, Yoshioka T, Takeda R, Sugawara A, Kuwahara T,Saleem MA, et al: Redistribution of connexin43 expression in glomerular podocytes predicts poor renal prognosis in patients with type 2 diabetes and overt nephropathy. Nephrol Dial Transplant 21: 2472-2477, 2006.

11. Yang M, Wang B, Li M and Jiang B: Connexin 43 is involved in aldosterone-induced podocyte injury. Cell Physiol Biochem 34: 1652-1662, 2014.

12. Prakoura N, Kavvadas P and Chadjichristos CE: Connexin 43: A new therapeutic target against chronic kidney disease. Cell Physiol Biochem 49: 985, 2018.

13. Murphy SF, Varghese RT, Lamouille S, Guo S, Pridham KJ, Kanabur P, Osimani AM, Sharma S, Jourdan J, Rodgers CM, et al: Connexin 43 inhibition sensitizes chemoresistant glioblastoma cells to temozolomide. Cancer Res 76: 139-149, 2016.
14. Zhang A, Han Y, Wang B, Li S and Gan W: Beyond gap junction channel function: The expression of $\mathrm{Cx} 43$ contributes to aldosterone-induced mesangial cell proliferation via the ERK1/2 and PKC pathways. Cell Physiol Biochem 36: 1210-1222, 2015.

15. Wang B, Xu X, He X, Wang Z and Yang M: Berberine improved aldo-induced podocyte injury via inhibiting oxidative stress and endoplasmic reticulum stress pathways both in vivo and in vitro. Cell Physiol Biochem 39: 217-228, 2016.

16. Bejarano E, Yuste A, Patel B, Stout RF Jr, Spray DC and Cuervo AM: Connexins modulate autophagosome biogenesis. Nat Cell Biol 16: 401-414, 2014.

17. Kim YC and Guan KL: mTOR: A pharmacologic target for autophagy regulation. J Clin Invest 125: 25-32, 2015.

18. Lichtenstein A, Minogue PJ, Beyer EC and Berthoud VM: Autophagy: A pathway that contributes to connexin degradation. J Cell Sci 124: 910-920, 2011.

19. Martins-Marques T, Catarino S, Zuzarte M, Marques C, Matafome P,Pereira P and Girão H: Ischaemia-induced autophagy leads to degradation of gap junction protein connexin43 in cardiomyocytes. Biochem J 467: 231-245, 2015.

20. Li C, Ye L, Yang L, Yu X, He Y, Chen Z, Li L and Zhang D: Rapamycin promotes the survival and adipogenesis of ischemia-challenged adipose derived stem cells by improving autophagy. Cell Physiol Biochem 44: 1762-1774, 2017.

21. Kimura T, Takabatake Y, Takahashi A and Isaka Y: Chloroquine in cancer therapy: A double-edged sword of autophagy. Cancer Res 73: 3-7, 2013.

22. Yasuda-Yamahara M, Kume S, Tagawa A, Maegawa H and Uzu T: Emerging role of podocyte autophagy in the progression of diabetic nephropathy. Autophagy 11: 2385-2386, 2015.

23. Liu M, Liang K, Zhen J, Zhou M, Wang X, Wang Z, Wei X, Zhang Y, Sun Y, Zhou Z, et al: Sirt6 deficiency exacerbates podocyte injury and proteinuria through targeting Notch signaling. Nat Commun 8: 413, 2017.

24. Kim SN, Kwon HJ, Im SW, Son YH, Akindehin S, Jung YS, Lee SJ, Rhyu IJ, Kim IY, Seong JK, et al: Connexin 43 is required for the maintenance of mitochondrial integrity in brown adipose tissue. Sci Rep 7: 7159, 2017.

25. Kroemer G: Autophagy: A druggable process that is deregulated in aging and human disease. J Clin Invest 125: 1-4, 2015.

26. Choi AM, Ryter SW and Levine B: Autophagy in human health and disease. N Engl J Med 368: 651-662, 2013.

27. Wang B, Ding W, Zhang M, Li H, Guo H, Lin L, Chen J and $\mathrm{Gu}$ Y: Role of FOXO1 in aldosterone-induced autophagy: A compensatory protective mechanism related to podocyte injury. Oncotarget 7: 45331-45351, 2016.

28. Haefliger JA, Demotz S, Braissant O, Suter E, Waeber B, Nicod P and Meda P: Connexins 40 and 43 are differentially regulated within the kidneys of rats with renovascular hypertension. Kidney Int 60: 190-201, 2001.

29. Laplante M and Sabatini DM: mTOR signaling in growth control and disease. Cell 149: 274-293, 2012.

30. Shimobayashi M and Hall MN: Making new contacts: The mTOR network in metabolism and signalling crosstalk. Nat Rev Mol Cell Biol 15: 155-162, 2014.

31. Gödel M, Hartleben B, Herbach N, Liu S, Zschiedrich S, Lu S, Debreczeni-Mór A, Lindenmeyer MT, Rastaldi MP, Hartleben G, et al: Role of mTOR in podocyte function and diabetic nephropathy in humans and mice. J Clin Invest 121: 2197-2209, 2011.

32. Cinà DP, Onay T, Paltoo A, Li C, Maezawa Y, De Arteaga J, Jurisicova A and Quaggin SE: Inhibition of MTOR disrupts autophagic flux in podocytes. J Am Soc Nephrol 23: 412-420, 2012.

33. Klionsky DJ, Abdelmohsen K, Abe A, Abedin MJ, Abeliovich $\mathrm{H}$, Acevedo Arozena A, Adachi H, Adams CM, Adams PD, Adeli K, et al: Guidelines for the use and interpretation of assays for monitoring autophagy (3rd edition). Autophagy 12: 1-222, 2016.

This work is licensed under a Creative Commons Attribution-NonCommercial-NoDerivatives 4.0 International (CC BY-NC-ND 4.0) License. 